\title{
Corela
}

Cognition, représentation, langage

HS-20 | 2016

L'Implicite

\section{Pragmatique et compréhension du langage : comment évaluer les inférences en jeu?}

\section{Virginie Laval, Sandrine Gil et Jamila Hattouti}

\section{OpenEdition}

\section{Journals}

Édition électronique

URL : http://journals.openedition.org/corela/4730

DOI : $10.4000 /$ corela.4730

ISSN : $1638-573 \mathrm{X}$

\section{Éditeur}

Cercle linguistique du Centre et de I'Ouest - CerLICO

\section{Référence électronique}

Virginie Laval, Sandrine Gil et Jamila Hattouti, « Pragmatique et compréhension du langage : comment évaluer les inférences en jeu ? ", Corela [En ligne], HS-20 | 2016, mis en ligne le 07 décembre 2016, consulté le 19 avril 2019. URL : http://journals.openedition.org/corela/4730 ; DOI : 10.4000/ corela.4730

Ce document a été généré automatiquement le 19 avril 2019

\section{(i) (2)}

Corela - cognition, représentation, langage est mis à disposition selon les termes de la licence Creative Commons Attribution - Pas d'Utilisation Commerciale - Partage dans les Mêmes Conditions 4.0 International. 


\title{
Pragmatique et compréhension du langage : comment évaluer les inférences en jeu?
}

\author{
Virginie Laval, Sandrine Gil et Jamila Hattouti
}

\section{Introduction}

1 La maîtrise des aspects formels du langage ne fournit qu'une vision partielle du développement des capacités langagières des enfants: elle renseigne sur la seule acquisition du système linguistique, sans rendre compte de la capacité des enfants à utiliser d'autres indices qui relèvent de la situation de communication. En effet, comprendre ce que dit l'autre n'est pas toujours une affaire exclusive de vocabulaire ou de grammaire, il faut aussi être en mesure de prendre en compte d'autres éléments tout aussi importants dans la compréhension du langage comme le contexte, le ton de la voix, l'expression faciale du locuteur, etc. La maîtrise complète d'une langue en usage implique donc la maîtrise de la relation entre énoncé et situation de communication. Cette relation, étudiée par le courant de la pragmatique, fait partie des compétences nécessaires à la communication au même titre que la maîtrise des aspects formels du langage (Laval, Aguert \& Gil, 2012 ; Laval \& Ryckebusch, 2010).

2 La pragmatique étudie les habiletés d'un individu à comprendre et/ou exprimer les intentions de communications par référence à une situation de communication (Gibbs, 1999 ; Laval \& Ryckebusch, 2010). Cette dernière constitue ainsi le cadre fondamental de l'investigation pragmatique : tout élément constitutif de la situation peut permettre des inférences sur l'intention de communication d'un locuteur. L'inférence sociale est au cœur des préoccupations des chercheurs en pragmatique, dont l'objectif est de mesurer l'impact d'un ensemble varié d'indices illocutoires sur la compréhension. Ces indices illocutoires peuvent se situer dans le comportement communicatif du locuteur, qu'ils soient directement linguistiques (comme inférer d'une marque morphologique 
structurale - comme le vouvoiement - des informations sur la relation qui unit les interlocuteurs), qu'ils soient

paralinguistiques (inférer que le locuteur n'est pas sincère sur la base de sa prosodie émotionnelle) ou qu'ils soient extralinguistiques (inférer du contexte situationnel que le locuteur est ironique). L'inférence sociale, nécessaire pour comprendre le message de son interlocuteur, permet de rendre compte de la diversité des indices illocutoires disponibles au sein d'une situation de communication (Martin \& McDonald, 2003). L'objectif de cette recherche est de proposer un protocole d'évaluation, issu de la recherche expérimentale, permettant de focaliser sur le rôle des inférences contextuelles et sémantiques dans la compréhension d'un énoncé produit dans une situation de communication.

4 Le protocole élaboré mobilise une forme particulière du langage non littéral: les expressions idiomatiques, généralement définies comme des locutions stéréotypées dont la signification est conventionnelle, et pas nécessairement déductible de la signification des mots qui la composent (Gibbs, 1994 ; Marquer, 2005). Il ne suffit pas, par exemple, de connaître le sens des mots « changer » et « disque » pour appréhender le sens idiomatique de l'expression "changer de disque » («parler d'autre chose »). Certaines expressions idiomatiques sont ambigües, c'est-à-dire qu'elles peuvent donner lieu à deux interprétations possibles - littérale et idiomatique - (par exemple, «les carottes sont cuites), et d'autres, au contraire, sont dépourvues d'acceptation littérale et n'ont qu'une seule interprétation idiomatique possible (par exemple, "être sur son trente-et-un »). Loin d'appartenir spécifiquement à un registre de langage peu utilisé, tel le registre soutenu, les expressions idiomatiques sont fréquentes dans nos conversations quotidiennes (Gibbs, 1994 ; Kerbel \& Grunwell, 1997; Weil-Barrais, 2004) . Comprendre une expression idiomatique est néanmoins une tâche difficile puisqu'on ne peut les considérer comme de simples produits des règles syntaxiques (Mettre cartes sur table) et sémantiques (Etre sur son trente-et-un). Elles exigent de l'auditeur qu'il soit en mesure de jouer avec différents niveaux d'interprétation du langage en relation avec le contexte de communication, et constitue à ce titre un outil de mesure efficace de l'inférence sociale (Weil-Barrais, 2004).

5 La compréhension des expressions idiomatiques a fait l'objet de nombreuses recherches en psychologie du développement, qui ont conduit à un ensemble conséquent et riche de connaissances. Le contexte joue un rôle fondamental dans la compréhension des expressions idiomatiques, y compris à l'adolescence (Cacciari \& Levorato, 1989; Cailles \& Lesourn, 2008 ; Cain, Oakhill \& Lemmon, 2005 ; Cain, Towse, \& Knight, 2009 ; Gibbs, 1987, 1991 ; Laval, 2003 ; Levorato \& Cacciari, 1992 ; Nippold \& Martin, 1989; Qualls, O’Brien, Blood, \& Hammer, 2003). Il ressort de ces travaux que l'inférence contextuelle apparait essentielle pour comprendre une expression idiomatique. De la même façon, le degré de transparence d'une expression correspond à un autre élément favorisant largement la compréhension des idiomes (Cain, et al., 2009; Gibbs, 1987, 1991; Levorato \& Cacciari, 1995, 1999 ; Nippold \& Rudzinski, 1993 ; Nippold \& Taylor, 1995, 2002 ; Nippold, Taylor \& Baker, 1996). Le degré de transparence est une propriété intrinsèque à l'idiome (Nippold \& Taylor, 2002). Il s'agit du lien qui existe entre les mots qui composent l'expression et l'interprétation. Par exemple, "sonner l'alarme » est une expression transparente, alors que « tailler une bavette « est une expression opaque. Les recherches ont montré que cette capacité d'analyse sémantique augmente avec l'âge, y compris à l'adolescence. Ainsi, l'inférence contextuelle et l'inférence sémantique constituent les deux processus 
nécessaires à la compréhension des idiomes, ces deux processus évoluant considérablement entre 5-6 ans et l'adolescence. S'il ne fait aucun doute que ces deux processus sont bien impliqués dans la compréhension, aucune des recherches précédentes n'a réellement testé la compréhension au sein de la situation de communication. La compréhension est principalement testée sur la base d'expressions idiomatiques insérés dans un environnement textuel (par écrit) ou lu par l'expérimentateur, plaçant davantage les participants dans une situation d'observateur que dans une situation d'interlocuteur.

\section{Présentation de l'étude}

Dans ce contexte, l'objectif de notre étude est de proposer un paradigme expérimental permettant d'étudier spécifiquement les processus impliqués dans la compréhension d'un énoncé produit dans une situation de communication. Par ailleurs, pour impliquer le participant dans l'interaction, nous avons construits des scénarii filmés reproduisant une situation d'interaction entre deux personnages, le participant étant toujours celui à qui s'adresse le locuteur du film, le plaçant ainsi comme interlocuteur principal de la scène.

7 Par ailleurs, l'étude de la compréhension des processus en jeu dans la compréhension d'un idiome ne peut réellement se faire que si l'on prend en compte les résultats contradictoires obtenus dans les recherches antérieures sur le rôle de la familiarité de l'idiome, défini par la fréquence d'usage (Laval, 2003 ; Levorato \& Cacciari, 1999 ; Nippold \& Taylor, 2002). Sur ce point, les recherches antérieures ont permis de montrer toute la difficulté à construire un matériel expérimental homogène du point de vue de la familiarité de l'idiome, que l'on travaille dans une perspective développementale ou chez l'adulte. Il est fort probable que les méthodes de mesure du degré de familiarité des idiomes, basées la plupart du temps sur des épreuves de jugement du degré de familiarité, ne soient pas efficaces (Laval, Laval \& Eme, 2014). Or, l'étude des processus en jeu dans la compréhension des idiomes implique un rôle central à la question de la familiarité de l'idiome : si les expressions idiomatiques sont connues des participants, on ne mesure pas la qualité inférentielle dans le processus de compréhension, mais la capacité des participants à retrouver dans leur lexique mental la signification conventionnelle de l'idiome. C'est pour cette raison que nous avons repris à notre compte la méthodologie originale utilisée par Cain et al. $(2005,2009)$, qui consiste à utiliser des idiomes traduits de langues étrangères et qui n'ont donc pas d'équivalents «mot à mot » dans la langue française.

Le protocole expérimental ainsi constitué devrait permettre de mettre à jour les deux processus nécessaires à la compréhension des Idiomes: l'inférence contextuelle et l'inférence sémantique. Il présente l'avantage de mesurer la compréhension des idiomes dans une situation plus écologique, sur la base de scénarii filmés impliquant le participant au cœur de l'interaction, et permet aussi de s'assurer que les idiomes à comprendre ne sont pas connus des participants, les incitant nécessairement à déclencher des inférences pour donner du sens à l'idiome. Avant d'être utilisé chez l'enfant et l'adolescent, nous avons souhaité le tester auprès d'un groupe d'adultes. 


\section{Eléments de méthodologie}

\section{Participants}

9 Cinquante-cinq adultes participants (âge moyen : 22 ans 4 mois) ont participé à cette recherche. Les participants, tous de langue maternelle française, étaient des étudiants de l'université de Poitiers.

\section{Matériel}

10 Le matériel est composé de petits scénarii filmés. Un ordinateur PC muni du logiciel Powerpoint permet la présentation des films. Chaque film est composé d'images fixes et d'une vidéo dynamique, d'une voix-off et d'une voix on-line. La voix-off a été enregistrée par une femme de langue maternelle française. La voix on-line est celle de l'acteur ou l'actrice principal(e) du scénario. Des carnets de passation ont été fournis aux participants pour recueillir leurs réponses par écrit.

11 Les films varient en fonction du contexte situationnel. Chaque idiome a été inséré systématiquement dans deux conditions : dans un contexte idiomatique, induisant le sens de l'expression, et en dehors de tout contexte.

\section{Contexte Idiomatique}

Dans la condition contexte idiomatique, chaque film est composé de trois parties. La première partie permet de poser le lieu où se déroule la scène, lieu qui est représenté par une image fixe de couleur sépia. La deuxième partie, qui prend la forme d'une vidéo dynamique aux couleurs naturelles, permet de poser clairement le contexte de production de l'idiome, induisant systématiquement une interprétation conventionnelle de l'expression. La troisième partie correspond à la production de l'idiome. Le locuteur s'adresse systématiquement à la caméra, simulant ainsi une interaction avec le participant. Les 12 idiomes expérimentaux ${ }^{1}$ sont non connus des participants, dans la mesure où ils sont traduits de langues étrangères et qu'ils n'ont pas d'équivalents « mot à mot» en français. Les idiomes sont pour moitié, opaques et pour l'autre moitié, transparents. Le Tableau 1 spécifie le principe général de déroulement de chaque scénario en contexte idiomatique à travers un exemple d'idiome opaque « lâcher le pigeon » 

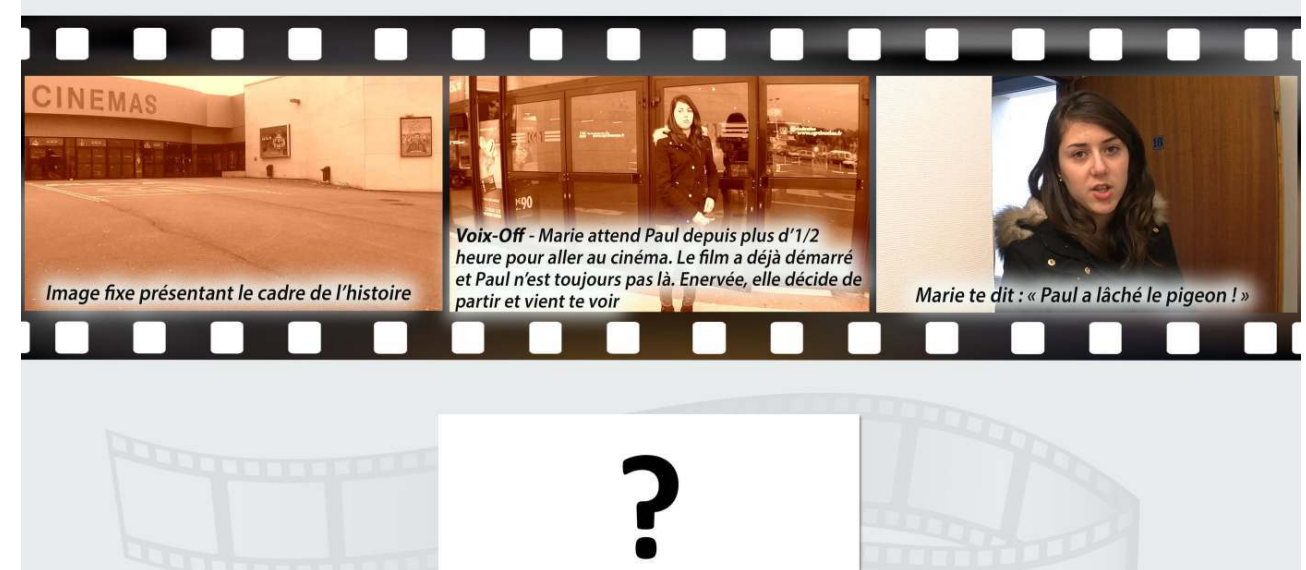

Que veut dire Marie?

(c) CeRCA

\section{Hors Contexte}

Dans la condition hors contexte, les parties 1 et 2 des scénarii en contexte idiomatique ont été supprimées. Les films, dans la condition hors contexte, sont construits à partir de la seule extraction de la troisième partie correspondant à la production de l'idiome. Ainsi l'expression idiomatique est produite à l'identique (prosodie, expression faciale, regard, etc.) dans les deux conditions. Le Tableau 2 spécifie le principe général de déroulement de chaque scénario dans la condition hors contexte à travers un exemple d'idiome opaque « lâcher le pigeon ". 


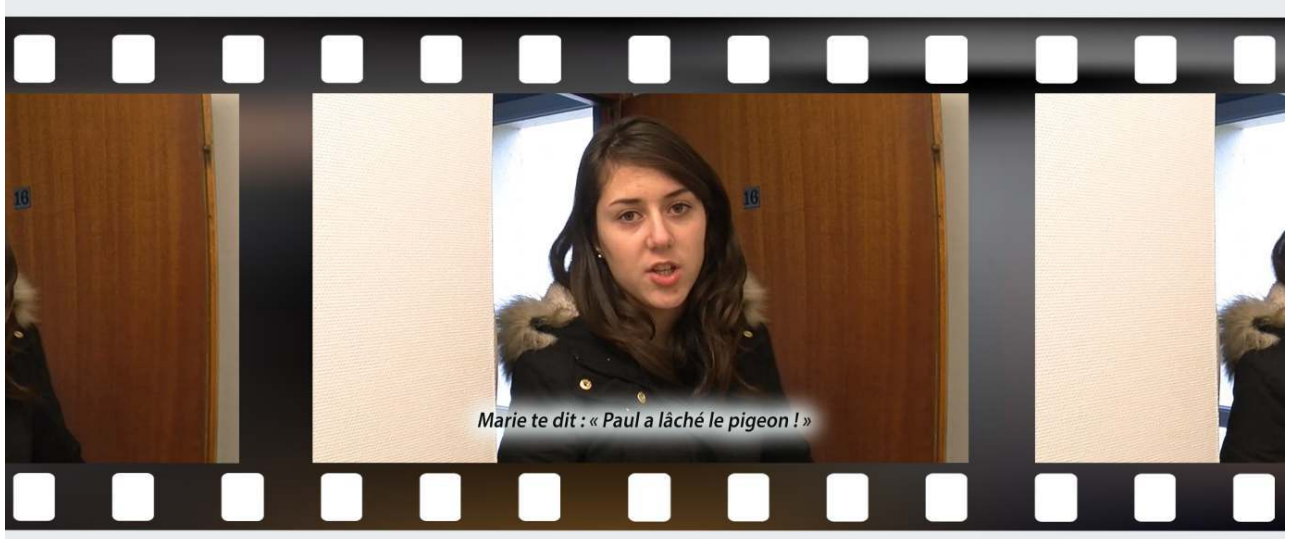

\section{Procédure}

La compréhension est testée à partir d'une tâche d'explication verbale, au cours de laquelle les adultes avaient à fournir l'interprétation idiomatique de l'expression présentée. Nous avons opté pour cette tâche d'explication verbale, largement utilisée dans les recherches antérieures (Cain et al., 2005 ; Gibbs, 1991 ; Levorato \& Cacciari, 1995 ; Nippold \& Martin, 1989; Nippold \& Rudzinski, 1993) pour deux raisons principales. Premièrement, dans une épreuve de choix, lorsque le participant doit choisir parmi plusieurs possibilités, les options proposées peuvent assez facilement biaiser la réponse du participant, même lorsque les options ont été construites avec la plus grande rigueur scientifique (Nippold \& Taylor, 1995). Ainsi, pour mesurer la compréhension en contexte, il est souvent très difficile, voire impossible, de faire cohabiter au sein d'un même ensemble de propositions une option idiomatique et une option littérale, lorsque les deux interprétations ne se recouvrent pas du tout. Par exemple, l'option littérale pour l'expression «mettre du beurre dans les épinards » est difficile à opérationnaliser et à rendre plausible dans un contexte orientant vers une interprétation idiomatique de l'expression (Cain et al., 2009). La seconde raison, liée à la première, est que la tâche d'explication verbale, certes plus difficile que l'épreuve de choix multiple (Ackerman, 1982 ; Gibbs, 1987, 1991 ; Nippold \& Martin, 1989 ; Nippold \& Taylor, 1995 ; Nippold, Taylor \& Baker, 1996), présente néanmoins l'avantage de ne pas contraindre la réponse du participant, et constitue ainsi un bon indicateur des stratégies de compréhension mises en œuvre par les participants (Cain et al., 2005). 


\section{Déroulement} condition hors contexte, puis 15 jours après, dans un contexte idiomatique. La passation est semi-collective : l'expérimentation a eu lieu par petits groupes de 5, et les réponses des participants sont individuelles. Lors de chaque phase, les participants visionnent l'ensemble des 12 films diffusés sur un écran à l'aide d'un vidéoprojecteur. Après la visualisation de chaque film, les participants répondent individuellement et par écrit à la question suivante : « que veut dire [prénom du locuteur] ?» (cf. Tableaux 1 et 2).

\section{Phase 1 : Idiomes hors contexte}

Avant la visualisation des idiomes hors contexte, la consigne expliquant la tâche à réaliser est présentée aux participants à l'écran et lue oralement par l'expérimentateur. La consigne pour les idiomes hors contexte est la suivante : «Vous allez assister à la projection de plusieurs petits films, dans lesquels vous allez être impliqués. À chaque fois, la personne va vous dire quelque chose. Vous allez devoir dire ce que la personne a voulu vous faire comprendre. Pour cela, après chaque film, vous devez répondre aux questions sur votre carnet papier: chaque film porte un numéro (ex: Hist 1). Ecoutez et regardez bien ce qui se passe dans chaque film car vous ne les verrez qu'une seule fois. On va essayer avec deux exemples. ».

Phase 2 : Idiomes en contexte idiomatique

Les idiomes en contexte sont présentés quinze jours après la première phase dans les mêmes conditions, mais avec la consigne suivante: "Comme la dernière fois, vous allez assister à la projection de plusieurs petits films, dans lesquels vous devez être impliqués. À chaque fois, la personne va vous dire quelque chose. Mais cette fois-ci, vous allez vous retrouver avec d'autres personnes dans différentes situations. Il faut donc que vous imaginiez bien participer à l'histoire. À la fin de chaque histoire, vous allez devoir dire ce que la personne a voulu vous faire comprendre. Ecoutez et regardez bien ce qui se passe dans chaque film car vous ne les verrez qu'une seule fois. On va essayer avec deux exemples. ».

\section{Analyse et résultats}

\section{Codage des données}

Les explications des idiomes ont été classées en 6 catégories selon la classification utilisée par Cain et al. (2005). Le Tableau 3 définit chaque catégorie et donne des exemples pour chacune d'entre elles. Deux juges indépendants ont réalisé le codage des réponses. Nous avons obtenu un taux d'accord inter-juges de $93 \%$ hors contexte et de $94 \%$ en contexte idiomatique. Un point a été attribué pour les réponses idiomatiques partielles et deux points pour les réponses idiomatiques afin d'obtenir un score de compréhension idiomatique par situation expérimentale. Aucun point n'était attribué pour toutes les autres réponses Ainsi, le score maximum de compréhension pour un participant et par case expérimentale était de 12 points (6 idiomes par case expérimentale). 
Exemple d'idiome: "Lâcher le pigeon " (Signification idiomatique: ne pas se rendre à un rendez-vous)

Histoire : « Marie attend Paul depuis plus d'une demi-heure pour aller au cinéma. Le film a déjà démarré et Paul n'est toujours pas là. Enervée elle décide de partir et vient te voir.

Marie te dit : «Paul a lâché le pigeon!»

Que veut dire Marie?

\begin{tabular}{|c|c|c|}
\hline Catégorie & $\begin{array}{l}\text { Exemple } \\
\text { hors contexte }\end{array}$ & $\begin{array}{l}\text { Exemple en } \\
\text { contexte } \\
\text { idiomatique }\end{array}$ \\
\hline $\begin{array}{l}\text { Réponse idiomatique : la réponse du participant indique } \\
\text { une compréhension explicite du sens figuré de } \\
\text { l'expression. La réponse peut correspondre à une } \\
\text { paraphrase ou à une conséquence du sens idiomatique. }\end{array}$ & $\begin{array}{l}\text { Il lui a posé un } \\
\text { lapin à leur } \\
\text { rendez-vous }\end{array}$ & $\begin{array}{l}\text { Paul n'est pas } \\
\text { venu, il m'a posé } \\
\text { un lapin }\end{array}$ \\
\hline $\begin{array}{l}\text { Réponse idiomatique partielle : la réponse du participant } \\
\text { indique une compréhension partielle du sens figuré de } \\
\text { l'expression. }\end{array}$ & Paul n'est pas là & Il m’a oublié \\
\hline $\begin{array}{l}\text { Réponse sans lien idiomatique : le participant cherche à } \\
\text { donner une interprétation figurée qui n'a pas de lien avec } \\
\text { le sens idiomatique de l'expression (ni avec le sens } \\
\text { littéral). }\end{array}$ & $\begin{array}{l}\text { Qu'elle a le } \\
\text { pigeon, qu'elle } \\
\text { n'a pas le moral }\end{array}$ & $\begin{array}{l}\text { Que Paul a } \\
\text { révélé le secret }\end{array}$ \\
\hline $\begin{array}{l}\text { Réponse littérale: la réponse du participant reflète la } \\
\text { signification concrète du sens des mots de l'expression } \\
\text { ou est en lien avec l'interprétation littérale : il peut s'agir } \\
\text { d'une paraphrase du sens littéral, d'une conséquence ou } \\
\text { d'une cause. }\end{array}$ & $\begin{array}{lr}\text { Le pigeon } & \text { qu'on } \\
\text { tenait } & \text { en } \\
\text { captivité } & \text { a été } \\
\text { relâché } & \text { par } \\
\text { Paul.» } & \end{array}$ & $\begin{array}{l}\text { Qu'il a laissé la } \\
\text { cage ouverte }\end{array}$ \\
\hline Répétition : la réponse est une répétition de l'expression & $\begin{array}{l}\text { Paul a lâché le } \\
\text { Pigeon }\end{array}$ & $\begin{array}{l}\text { Paul a lâché le } \\
\text { Pigeon }\end{array}$ \\
\hline $\begin{array}{l}\text { Autre : le participant répond « Je ne sais pas » ou quelque } \\
\text { chose de similaire. Le participant } \\
\text { fournit une réponse inintelligible. }\end{array}$ & Je n'ai pas d'idée & Je ne sais pas \\
\hline
\end{tabular}

\section{Résultats}

La variable dépendante "score de compréhension idiomatique» a été traitée à l'aide d'une analyse de la variance à 2 facteurs : Type de contexte (2) x Degré de transparence (2). La Figure 1 indique le score de compréhension idiomatique en fonction du type de contexte et du degré de transparence des expressions. Les principaux résultats sont les suivants. 
'effet $\mathrm{du}$ type de contexte est significatif $(F(1,54)=590.97, p<.0001)$, les adultes obtiennent un score de compréhension idiomatique plus élevé dans la condition « contexte idiomatique » $(M=10.24, E . T=1.34)$ que dans la condition « hors contexte » $(M$ $=4.80, E . T=3.59)$. L'effet du degré de transparence est significatif $(F(1,54)=142.48, p$ $<.0001$ ), les expressions idiomatiques transparentes entrainent un score de compréhension idiomatique plus élevé $(M=9, E . T=2.41)$ que les expressions idiomatiques opaques $(M=6.05, E . T=4.40)$. On observe un effet d'interaction entre le type de contexte et le degré de transparence $(F(1,54)=185.75, p<.0001)$. Dans la condition «hors contexte", les expressions idiomatiques transparentes entrainent un score de compréhension idiomatique plus important $(M=7.63, E . T=2.48$ que les expressions idiomatiques opaques $(M=1.98, E . T=1.91)(F(1,54)=232.5, p<.0001)$. Cette différence n'existe pas dans la condition « contexte idiomatique » $\left(M_{\text {transparente }}=10.36, E . T=1.36 ; M\right.$ opaque $=10.12$, E.T $=1.32$ ).

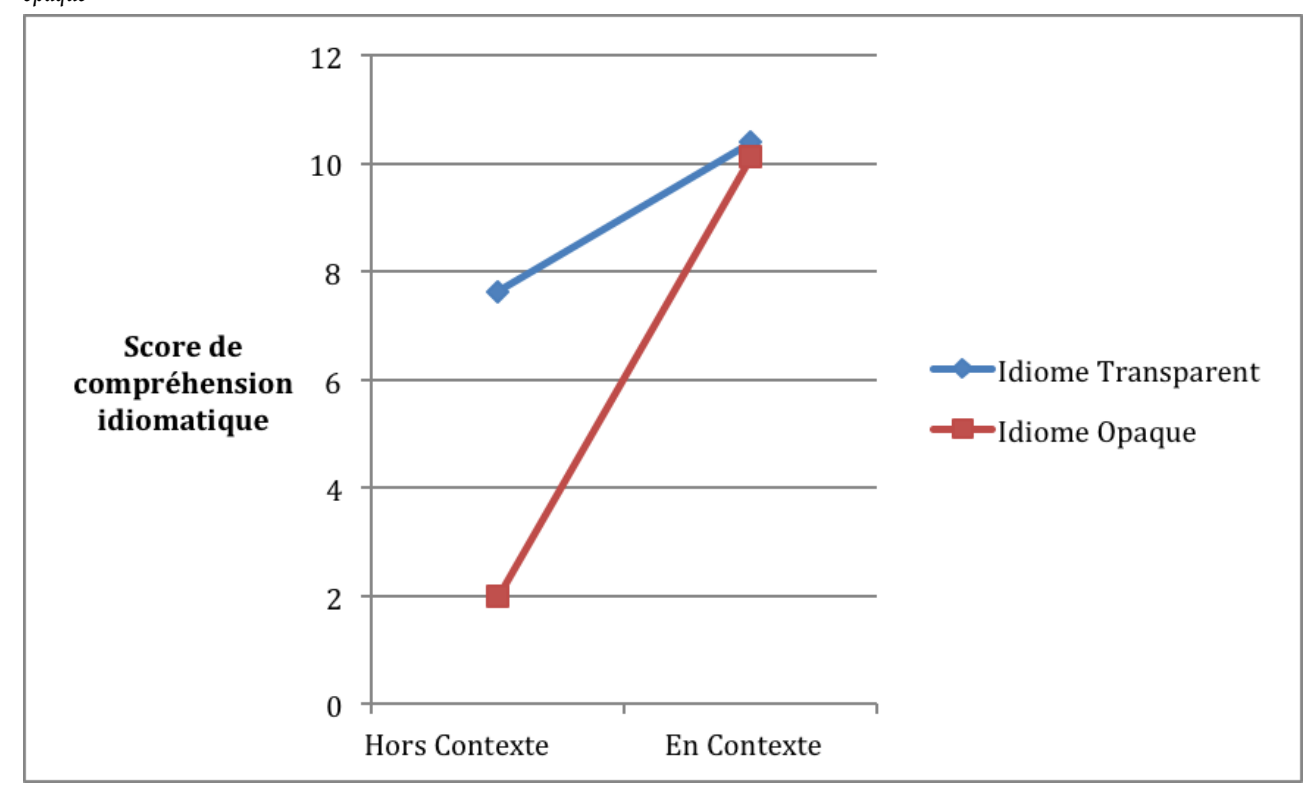

Figure 1 - Score de compréhension idiomatique en fonction du type de contexte et du degré de transparence des expressions.

\section{Conclusion}

21 L'objectif de cette recherche était de proposer un protocole d'évaluation, issu de la recherche expérimentale, permettant de mesurer le rôle des inférences contextuelles et des inférences sémantiques dans la compréhension des expressions idiomatiques. Ce protocole d'évaluation était original au moins sur deux points : construit sur la base de scénarii filmés, il permet de tester la compréhension des idiomes dans des situations d'interaction plus écologiques que ce qui n'avait été fait dans les recherches antérieures (Gibbs, 1991 ; Levorato \& Cacciari, 1995 ; Nippold \& Martin, 1989 ; Nippold et Rudzinski, 1993, Cain et al., 2005), et permet aussi d'impliquer plus le participant dans l'interaction. Basé sur la compréhension d'idiomes traduits de langues étrangères, il permet de s'assurer que les idiomes n'étaient pas connus des participants et devaient, pour être compris, faire l'objet d'inférences (Cain et al., 2005, 2009).

Les résultats obtenus chez les participants adultes nous conduisent à affirmer que le protocole d'évaluation permet bien de mesurer les inférences en jeu dans la 
compréhension des idiomes: il permet, de façon objective, de mesurer l'inférence contextuelle et l'inférence sémantique impliquées dans le processus de compréhension. De fait, cette recherche apporte une réponse heuristique à la question des normes développementales des capacités pragmatique en compréhension. La prochaine étape sera donc de dresser le tableau développemental des processus en jeu dans la compréhension chez l'enfant et chez l'adolescent pour aboutir à des repères développementaux. Enfin, cette recherche apporte des pistes prometteuses en matière d'évaluation des capacités pragmatiques du langage. L'examen des capacités langagières de l'enfant, focalisé principalement sur l'évaluation des caractéristiques formelles du langage, inclut encore trop rarement une exploration systématique de ses capacités pragmatiques. Pour mieux comprendre les troubles du langage, il s'avère nécessaire de disposer d'outils cliniques évaluant les inférences pragmatiques. En mesurant de façon objective les inférences en jeu dans le processus de compréhension, notre outil constitue une réponse concrète à la question de l'évaluation des capacités pragmatiques.

\section{BIBLIOGRAPHIE}

Ackerman, B. P. (1982). On comprehending idioms: do children get the picture? Journal of Experimental Child Psychology, 33, 439-454.

Cacciari, C., \& Levorato, M. C. (1989). How children understand idioms in discourse. Journal of Child Language, 16(2), 387-405.

Caillies, S., \& Le Sourn-Bissaoui, S. (2008). Children's understanding of idioms and theory of mind development. Developmental Science, 11, 703-711.

Cain, K., Oakhill, J., \& Lemmon, K. (2005). The relation between children's reading comprehension level and their comprehension of idioms. Journal of Experimental Child Psychology, 90(1), 65-87.

Cain, K., Towse, A. S., \& Knight, R. S. (2009). The development of idiom comprehension: An investigation of semantic and contextual processing skills. Journal of Experimental Child Psychology, 102(3), 280-298.

Gibbs, R. W. (1987). Linguistic factors in children's understanding of idioms. Journal of Child Language, 14(3), 569-586.

Gibbs, R. W. (1991). Semantic analyzability in children's understanding of idioms. Journal of Speech \& Hearing Research, 34(3), 613-620.

Gibbs, R. W. (1994). The poetics of mind: Figurative thought, language, and understanding. New York, NY US: Cambridge University Press.

Gibbs, R. W. (1999). Interpreting What Speakers Say and Implicate. Brain and Language, 68(3), 466-485. doi:10.1006/brln.1999.2123

Kerbel, D., \& Grunwell, P. (1997). Idioms in the classroom: An investigation of language unit and mainstream teachers' use of idioms. Child Language Teaching and Therapy, 13(2): 113-23. 
Laval, V. (2003). Idiom comprehension and metapragmatic knowledge in French children. Journal of Pragmatics, 35(5), 723-739.

Laval, V., Aguert, M., \& Gil S. (2012). Pragmatique et compréhension du langage chez l'enfant : la question de l'évaluation. In P. Allain, G. Aubin \& D. Le Gall (Eds). Cognition sociale et Neuropsychologie (pp. 265-282). Marseille : SOLAL.

Laval, V., Laval, C., \& Eme, E. (2014). Expressions idiomatiques et compréhension : la question de l'évaluation de la familiarité des idiomes dans une perspective développementale, In S. Le SournBissaoui, G. Le Maner-Idrrissi, V. Dardier, E. Bonjour, \& A. Lacroix (Eds.) Développement et variabilités (pp. 197-212). Rennes : Presses Universitaires de Rennes.

Laval, V., \& Ryckebusch, C. (2010). Etudier la compréhension en situation d'interaction : l'exemple des actes de langage. In J. Bernicot, E.Veneziano, M. Musiol \& A. Bert-Erboul (Eds). Interactions verbales et acquisition du langage (pp. 261-282). Paris : l'Harmattan.

Levorato, M. C., \& Cacciari, C. (1992). Children's comprehension and production of idioms: The role of context and familiarity. Journal of Child Language, 19(2), 415-433.

Levorato, M. C., \& Cacciari, C. (1995). The effect of different tasks on the comprehension and production of idioms in children. Journal of Experimental Child Psychology, 60, 261-283.

Levorato, M. C., \& Cacciari, C. (1999). Idiom comprehension in children: Are the effects of semantic analysability and context separable? European Journal of Cognitive Psychology, 11(1), 51-66.

Marquer, P. (2005). L'organisation du lexique mental. Des "contraires" aux expressions idiomatiques. Paris: L'Harmattan

Martin, I., \& McDonald, S. (2003). Weak coherence, no theory of mind, or executive dysfunction? Solving the puzzle of pragmatic language disorders, Brain and Language, 85(3), 451-466

Nippold, M. A., \& Martin, S. T. (1989). Idiom interpretation in isolation versus context: A developmental study with adolescents. Journal of Speech \& Hearing Research, 32(1), 59-66.

Nippold, M. A., \& Rudzinski, M. (1993). Familiarity and transparency in idiom explanation: A developmental study of children and adolescents. Journal of Speech \& Hearing Research, 36(4), 728-737.

Nippol, M. A., \& Taylor, C. L. (1995). Idiom understanding in youth: futher examination of familiarity and transparency. Journal of Speech and Hearing Research, 38, 426-433.

Nippol, M. A., Taylor, C. L., \& Baker, J. M. (1996). Idiom understanding in Australian youth: a cross-cultural comparaison. Journal of Speech and Hearing Research, 39, 442-447.

Nippold, M. A., \& Taylor, C. L. (2002). Judgments of idiom familiarity and transparency: A comparison of children and adolescents. Journal of Speech, Language, and Hearing Research, 45 (2), 384-391.

Qualls, C. D., O’Brien, R. M., Blood, G. W., \& Hammer, C. S. (2003). Contextual variation, familiarity, academic literacy, and rural adolescents' idiom knowledge. Language, Speech, and Hearing Services in Schools, 34(1), 69-79.

Weil-Barrais, A. (2004). Les apprentissages scolaires. Rosny-sous-Bois : Bréal 


\section{NOTES}

1. Les 12 idiomes expérimentaux sont issus d'une étude pilote au cours de laquelle 14 adultes devaient juger du degré de transparence de 50 idiomes traduits de langue étrangère. Pour chaque idiome, ils devaient estimer sur une échelle en 5 points la force du lien entre le sens des mots qui composent l'expression et sa signification idiomatique. Les 6 idiomes les plus transparents et les 6 idiomes les plus opaques ont été retenus.

\section{RÉSUMÉS}

Cette recherche vise à proposer un outil informatisé d'évaluation, issu de la recherche expérimentale, permettant de mesurer les inférences en jeu dans la compréhension du langage en situation de communication. L'outil a été construit sur la base de scénarii filmés présentant des situations de communication et impliquant le participant au cœur de l'interaction : le locuteur s'adresse systématiquement à la caméra et produit une expression idiomatique qui n'existe pas dans la langue française (par exemple, lâcher le pigeon). Pour comprendre, le participant est obligé de mettre en œuvre des stratégies de compréhension : soit analyser les mots, soit utiliser le contexte, soit les deux. L'outil a été testé auprès d'un groupe d'adultes. Les résultats ont montré que l'outil permettait bien de mesurer les inférences contextuelles et les inférences sémantiques impliquées dans la compréhension.

The aim of this study was to suggest a computerised assessment system, stemming from experimental research, evaluating language inferences in the context of a situation of communication. The tool was composed of small movies presenting various situations of communication and placing the participant at the centre of the interaction: one character speaks directly to the camera (i.e. to the participant), producing an idiom. The idioms used were translated from others languages and have no French equivalents (e.g., to drop a pigeon). In order to understand the meaning the participant must use inferences from a semantic or a contextual analysis. The experimental design was tested with an adult population. Results demonstrated that both kind of inferences are necessary for language comprehension.

\section{INDEX}

Mots-clés : Pragmatique, Inférences sociales, Compréhension du langage, Outils d'évaluation, expressions idiomatiques

Keywords : Pragmatic, Social inferences, Language comprehension, Assessment tool, Idiomatic expression 


\section{AUTEURS}

VIRGINIE LAVAL

Université de Poitiers, Laboratoire CerCA, UMR 7295, CNRS

\section{SANDRINE GIL}

Université de Poitiers, Université de Tours, Laboratoire CerCA, UMR 7295, CNRS

JAMILA HATTOUTI

Laboratoire CerCA, UMR 7295, CNRS 